Case report

\title{
Bifid pancreas tail and superior horizontal pancreatic artery of Popova: an unusual duet
}

\author{
Serghei Covantev, Natalia Mazuruc, Olga Belic \\ State University of Medicine and Pharmacy "Nicolae Testemitanu", Chisinau, Republic of Moldova
}

Received 16 August 2017, Accepted 9 October 2017

(C) 2017, Covantev S., Mazuruc N., Belic O.

(C) 2017, Russian Open Medical Journal

Abstract: Anomalies and variants of development of the pancreas are relatively frequent. Bifid pancreas tail is a rare developmental variant an there are only a few case descriptions in the literature. There were also no reports of significant vascular variations associated with bifid pancreas tail. We present a case of a bifid pancreas tail associated with superior horizontal pancreatic artery of Popova encountered during an anatomical dissection of a 64 years old male cadaver with a detailed analysis of the vascularization of the gland and its ductal system morphology.

Keywords: bifid pancreas tail, superior horizontal pancreatic artery of Popova, pancreas blood supply.

Cite as Covantev S, Mazuruc N, Belic O. Bifid pancreas tail and superior horizontal pancreatic artery of Popova: an unusual duet. Russian Open Medical Journal 2018; 7: e0203.

Correspondence to Serghei Covantev. Address: 165, Stefan cel Mare si Sfant, Bd. MD-2004, Chişinau, Republic of Moldova. Tel.: +373 69948085.

E-mail: kovantsev.s.d@gmail.com.

\section{Introduction}

Pancreas is one of the most inaccessible organs, lying so deep within the abdominal cavity, makes it often difficult to confirm the diagnosis. Thus, management of pancreatic disease remains a major challenge even in the XXI century. The advances of the surgical field made possible procedures on the pancreas that are more complicated. This requires detailed knowledge of anatomy of the region and its possible anomalies to prevent misdiagnosis and intraoperative complications.

Anomalies and variants of development of the pancreas are relatively frequent. In general, there are three groups of pancreas anomalies. The first group includes the migration anomalies (ectopic pancreas and annular pancreas), the second group fusion anomalies (pancreas divisum) and the third group are anomalies that affect the number and form, or only the configuration of the main pancreatic duct (bifid ducts) [1]. Variations of forms, position, direction and vascularization of the pancreas are also frequently encountered, although are usually not included within these three categories.

Bifid pancreas tail is a rare developmental variant. There are only a few case descriptions of it in the literature. There were also no reports of significant vascular variations associated with bifid pancreas tail. The knowledge of such a case is important for diagnostic and surgical procedures, since it can lead to misdiagnosis in clinical practice.

\section{Case report}

We present a case of bifid pancreas tail and superior horizontal pancreatic artery of Popova in a male cadaver (64 years). The pancreas measured $14 \mathrm{~cm}$ in length and $3.5^{*} 2 * 2 \mathrm{~cm}$ in height at the level of the head, body and tail, respectively. Each tail of the pancreas measured $1.9 \mathrm{~cm}$ in length (Figures 1 and 2).

The superior horizontal pancreatic artery of Popova began from the splenic artery and ran along the superior border of the pancreas, but before entering deep into the gland received a tribute from the splenic artery forming an arcade at this level (Figure 3 ).

Within the pancreas, the artery divided in horizontal and descending branches. The horizontal branch further divided into two branches supplying a part of the body and the two tails of the pancreas. The descending branch vascularized the body and then emerged at the level of posterior and inferior part of the pancreas running on the dorsal pat of the gland until the head of the pancreas. During its course, it gave off small pancreatic branches. At the level of the body, it anatomized with the inferior pancreatic artery (Figure 4). The dorsal pancreatic artery gave of three branches: inferior pancreatic artery and two branches to the head of the pancreas. One of its major branches anatomized with the superior anterior pancreatic artery and the other with superior posterior pancreatic artery (Figure 5 ).

The main pancreatic duct at its beginning measured $0.5 \mathrm{~cm}$ and the accessory pancreatic duct $0.2 \mathrm{~cm}$. At the level of the tail, the duct divided into three branches: one drained the left tail, the other - the right tail and the third - the intermediate pancreatic tissue between the tails (Figure 6).

The spleen measured $7.4 * 4.3 \mathrm{~cm}$ in length and width, respectively. The splenic artery gave of one superior polar artery and ended up branching into two arteries of the first order. The inferior branch of the first order gave of an artery to the tail of the pancreas, which entered the gland between the two tails and anatomized with the branches of superior horizontal pancreatic artery of Popova (Figure 2). 


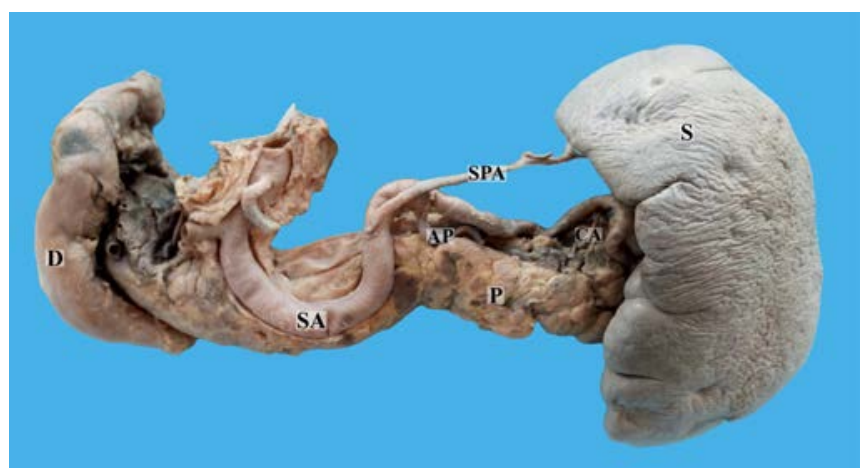

Figure 1. Pancreas and spleen with their blood supply.

D, duodenum; P, pancreas; S, spleen; SA, splenic artery; SPA, superior polar artery; CA, caudal artery; AP, artery of Popova.

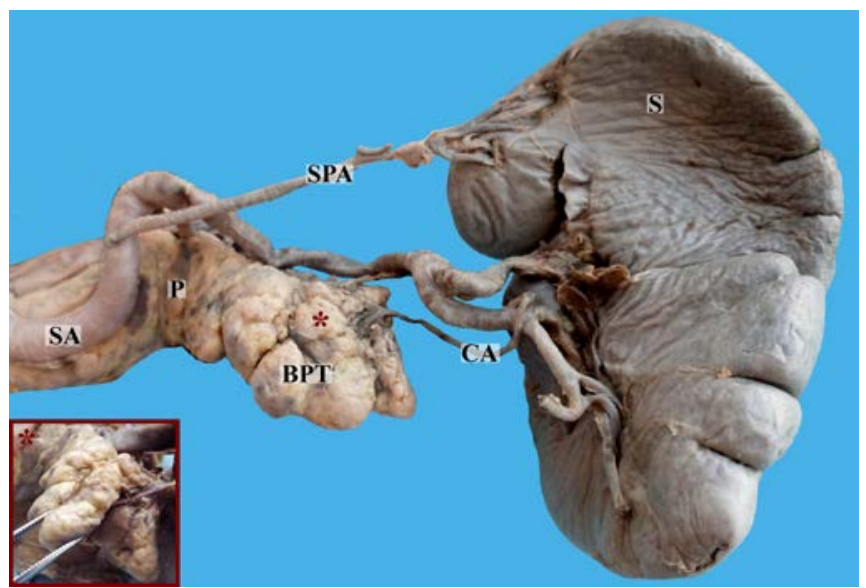

Figure 2. Fish tail pancreas.

P, pancreas; S, spleen; SA, splenic artery; SPA, superior polar artery; CA, caudal artery; BPT, bifid tail of the pancreas.

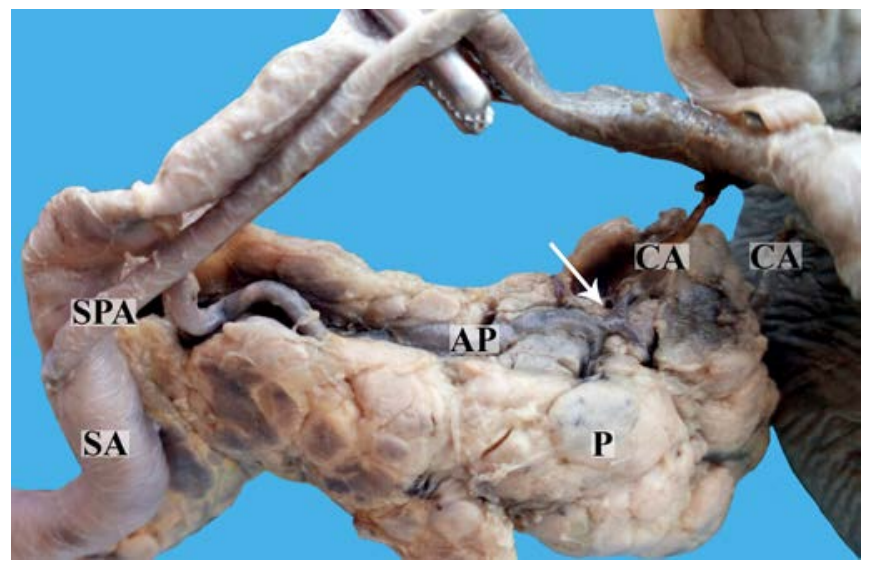

Figure 3. Trajectory of the artery of Popova.

SA, splenic artery; SPA, superior polar artery; CA, caudal artery; AP, artery of Popova. White arrow - anastomosis between the artery of Popova and the caudal artery.

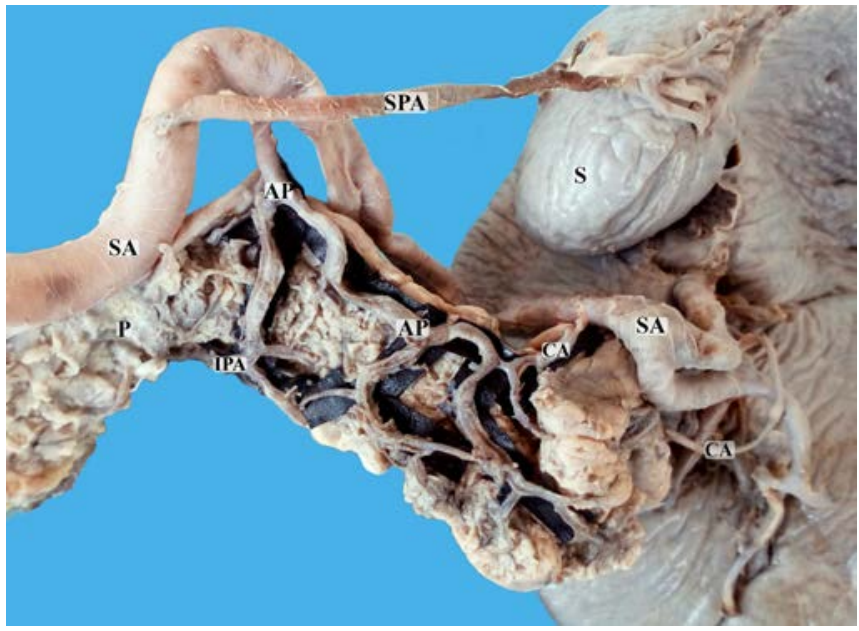

Figure 4. Blood supply to the tail of the pancreas.

$P$, pancreas; S, spleen; SA, splenic artery; SPA, superior polar artery; $C A$, caudal artery; AP, artery of Popova; IPA, inferior pancreatic artery.

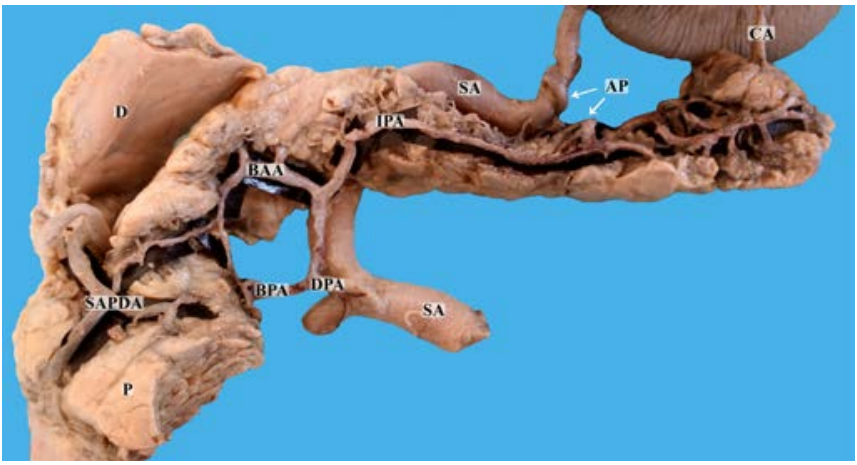

Figure 5. The dorsal pancreatic artery with its branches.

D, duodenum; P, pancreas; SA, splenic artery; CA, caudal artery; AP, artery of Popova; IPA, inferior pancreatic artery; DPA, dorsal pancreatic artery; $\mathrm{BAA}$, branch to the anterior archade of the head of pancreas; BPA, branch to the posterior archade of the head of pancreas; SAPDA, anterior superior pancreatic artery.

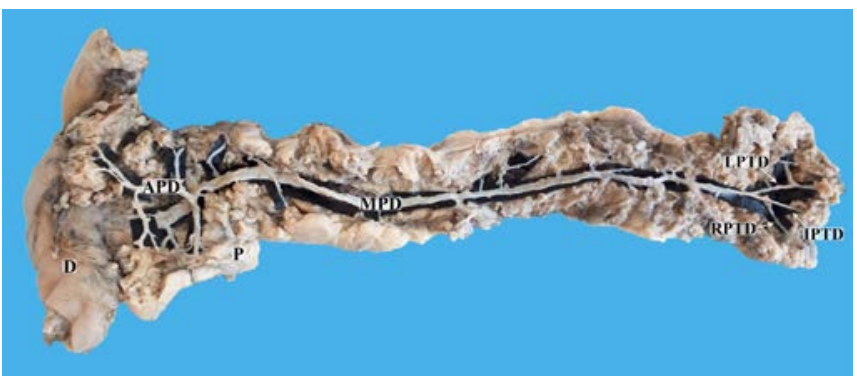

Figure 6. Pancreatic duct.

$D$, duodenum; P, pancreas; MPD, main pancreatic duct; APD, accessory pancreatic duct; LPTD, left pancreatic tail duct; RPTD, right pancreatic tail duct; IPTD, intermediate pancreatic tail duct. 


\section{Discussion}

Normal embryologic development of the pancreas is thought to begin approximately 5 weeks as two budlike outgrowths of mesoderm from the foregut that represent the ventral and dorsal buds. The dorsal bud grows more rapidly and rotates, and approximately 8 weeks after fertilization, the two parts unite to form the pancreatic gland. The ventral bud gives rise to the head and the main pancreatic duct. The other bud becomes the uncinate process, body and tail of the pancreas [2].

Often the only indication of the bifid pancreas tail (pancreas bifidum or fish tail pancreas) is the branching of the main pancreatic duct and can be seen on endoscopic retrograde cholangiopancreatography (ERCP) or magnetic resonance cholangiopancreatography [1]. In general, it is considered to be a benign variant of development with little or no clinical significance. Still, taking in consideration that it is rarely encountered it is hard to say if any pathological condition is tightly associated with it.

In a report of 650 ERCP, there were only 7 cases of bifid ductal system at the level of pancreas tail [3]. Thus around a bifid ductal system was encountered in $1.1 \%$ of cases. It should be noted though that the division of the ductal system at the level of the pancreas tail does not always indicate a bifid pancreas tail. On the opposite, the presence of bifid pancreas tail indicates a ductal bifurcation at this level. Although in our case, the main pancreatic duct branched into three terminal ducts: left pancreatic tail duct, right pancreatic tail duct and an intermediate pancreatic tail duct.

Bifid tail of the pancreas can be the reason for misdiagnosis and can presents as a tumor of the pancreas or adjacent to it organs [4]. There has also been a case report of a localized acute pancreatitis of one of the tails [5]. There are also localized processes that can affect the tail of the pancreas such as autoimmune pancreatitis, tumors and others that require differential diagnosis [6]. Besides ductal anomalies are linked with acute pancreatitis which makes pancreas bifidum also a reasonable candidate [7].

The artery of Popova is not a commonly accepted term; in fact, it is not the part of anatomical terminology. In 1910, Popova described a superior horizontal pancreatic artery, which was named after her and then unfortunately fell into oblivion for ages. The artery starts directly from the splenic artery and runs along the superior border of the body and tail of the pancreas [8].

The most comprehensive study of its incidence was conducted by Mosca and coworkers and demonstrated that it could be identified in $25.9 \%$ of cases on 1,015 angiographies. They have demonstrated that in $70.6 \%$ of cases the superior horizontal pancreatic artery was co-existing with the inferior pancreatic artery, with which it joined through several descending branches, and in $17.6 \%$ of cases, it could end anastomosing with the caudal pancreatic artery. Less frequently ( $11.7 \%$ of cases), the artery appeared as the only artery supplying the distal pancreas. In the majority of cases $(88.2 \%$ of cases), the superior horizontal pancreatic artery divided in a comb-like fashion giving off several descending branches; they distributed to the pancreas and/or ended anastomosing with the inferior pancreatic artery [9]. In our case, the artery was well developed and had several tributaries along its course from the splenic artery. It vascularized the major region of the distal part of the pancreas and ended up anastomosing with the inferior pancreatic artery.

\section{Conclusion}

We present a rare case of pancreas bifidum with a co-exiting superior horizontal pancreatic artery of Popova. We demonstrated how the artery can vascularize a major portion of the gland when it receives multiple tributaries from the splenic artery. This knowledge can be useful in surgical and diagnostic procedures.

Conflict of interest: none declared.

\section{References}

1. Dinter D, Lohr JM, Neff KW. Bifid tail of the pancreas: benign bifurcation anomaly. AJR Am J Roentgenol 2007; 189(5): W251-W253. https://dx.doi.org/10.2214/AJR.05.1453.

2. Pandol SJ. Pancreatic Embryology and Development. In: The Exocrine Pancreas. San Rafael (CA): Morgan \& Claypool Life Sciences, 2010. https://www.ncbi.nlm.nih.gov/books/NBK54135/.

3. Uomo G, Manes G, D'Anna L, Laccetti M, Di Gaeta S, Rabitti PG. Fusion and duplication variants of pancreatic duct system. Int J Pancreatol 1995; 17(1): 23-28. https://dx.doi.org/10.1007/BF02788355.

4. Kikuchi K, Nomiyama T, Miwa M, Harasawa S, Miwa T. Bifid tail of the pancreas: a case presenting as a gastric submucosal tumor. Am J Gastroenterol 1983; 78(1): 23-27. https://www.ncbi.nlm.nih.gov/pubmed/6849310.

5. Koyasu S, Isoda H, Nakase H, Kodama Y, Chiba T, Togashi K. Bifid tail of the pancreas with localized acute pancreatitis. Magn Reson Med Sci 2013; 12(4): 315-318. https://dx.doi.org/10.2463/mrms.2013-0033.

6. Taniguchi T, Seko S, Azuma K, Tamegai M, Nishida O, Inoue F, et al. Autoimmune pancreatitis detected as a mass in the tail of the pancreas. J Gastroenterol Hepatol 2000; 15(4): 461-464. https://dx.doi.org/10.1046/j.1440-1746.2000.02088.x.

7. Machado MA, Basseres T, Surjan RC, Makdissi FF. Laparoscopic partial pancreatectomy in a patient with pancreas trifurcation and recurrent acute pancreatitis. JOP J Pancreas 2016; S(1): 142-143. http://pancreas.imedpub.com/laparoscopic-partial-pancreatectomyin-a-patient-with-pancreas-trifurcation-and-recurrent-acutepancreatitis. php?aid=7879.

8. Popova AV. Contribution a l'étude de la distribution du système artériel dans le pancréas des enfants nés avant term. Arch Soc Sci Med Biol Montpellier 1910; 15: 139-144.

9. Mosca S, Di Gregorio F, Regoli M, Bertelli E. The superior horizontal pancreatic artery of Popova: a review and an anatomoradiological study of an important morphological variant of the pancreatica magna artery. Surg Radiol Anat 2014; 36(10): 1043-1049. https://dx.doi.org/10.1007/s00276-014-1276-8.

\section{Authors:}

Serghei Covantev - MD, Junior Researcher, Laboratory of Allergology and Clinical Immunology, State University of Medicine and Pharmacy "Nicolae Testemitanu", Chisinau, Republic of Moldova. http://orcid.org/0000-00017236-5699.

Natalia Mazuruc - MD student, Department of Human Anatomy, State University of Medicine and Pharmacy "Nicolae Testemitanu", Chisinau, Republic of Moldova. http://orcid.org/0000-0003-0610-5404.

Olga Belic - MD, PhD, Associate Professor, Department of Human Anatomy, State University of Medicine and Pharmacy "Nicolae Testemitanu", Chisinau, Republic of Moldova. 